Pacific Journal of Mathematics

ON GENERALIZED LIMITS AND LINEAR FUNCTIONAL 


\section{ON GENERALIZED LIMITS AND LINEAR FUNCTIONALS}

\section{ABRAham Robinson}

1. Introduction. Let $m$ be the space of bounded sequences of real numbers, $\sigma=\left\{s_{n}\right\}, n=1,2,3, \cdots$ under the norm $\|\sigma\|=\sup \left|s_{n}\right|$. Then $m$ includes the set of all convergent sequences of real numbers. Let $A=\left(a_{k n}\right), k, n=1,2,3, \cdots$ be a real Toeplitz-matrix, i.e. an infinite matrix of real numbers which satisfies the conditions

$$
\begin{array}{ll}
\lim _{k \rightarrow \infty} a_{k n}=0, & n=1,2, \cdots ; \\
\sum_{n=1}^{\infty}\left|a_{k n}\right|<M, &
\end{array}
$$

where $M$ is a positive number which is independent of $k$;

$$
\lim _{k \rightarrow \infty} \sum_{n=1}^{\infty} a_{k n}=1 \text {. }
$$

It is well-known that $A$ defines a regular method of summation. That is to say, if $\left\{s_{n}\right\}$ is a convergent sequence in $M$, with limit $s$, then the transform $\left\{t_{k}\right\}$ of $\left\{s_{n}\right\}$ by $A$ exists, where

$$
t_{k}=\sum_{n=1}^{\infty} a_{k n} s_{n}
$$

and $\left\{t_{k}\right\}$ converges to the same limit as $\left\{s_{n}\right\}$,

$$
\lim _{k \rightarrow \infty} t_{k}=\lim _{n \rightarrow \infty} s_{n}=s \text {. }
$$

If $\left\{s_{n}\right\}$ is an arbitrary element of $m$ then $\left\{t_{k}\right\}$ exists anyhow and may (or may not) converge to a limit, which is then called the $A$-limit of $\left\{s_{n}\right\}$. However, it has been shown by Steinhaus that for every Toeplitz-matrix $A$ there exists a sequence $\left\{s_{n}\right\}$ in $m$ which is not summed by $A$, i.e. such that $\left\{t_{k}\right\}$ does not converge (compare e.g. Ref. 2). Nevertheless the method of summation by infinite matrices is very useful in various branches of real and complex Function Theory. We shall call the limits obtained in this way Toeplitz-limits, to distinguish them from other types of generalized limits such as those discussed below.

A straightforward application of the Hahn-Banach extension theorem shows that there exist continuous linear functionals $F(x)$ defined on $m$ such that for every convergent sequence $\sigma=\left\{s_{n}\right\}$ in $m$, $F(\sigma)=\lim _{n \rightarrow \infty} s_{n}$. Such a functional will be called a Hahn-Banach-limit. 
In view of Steinhaus' theorem, mentioned above, a Hahn-Banach-limit cannot be a Toeplitz-limit, although every Toeplitz-limit can be extended to a Hahn-Banach-limit, again by applying the Hahn-Banach extension theorem.

If $F(x)$ is a Hahn-Banach-limit such that

1.6. (positivity) $s_{n} \geqq 0$ for $n=1,2, \cdots$ entails $F\left(\left\{s_{n}\right\}\right) \geqq 0$ and such that

1.7. (shift invariance) $F\left(\left\{s_{n}\right\}\right)=F\left(\left\{s_{n+1}\right\}\right)$ for all $\left\{s_{n}\right\} \in m-$ then $F(x)$ will be called a Mazur-Banach limit. It is known that there exist Mazur-Banach limits (compare ref. 1 or ref. 10).

In spite of their greater efficiency, the generalized limits which are defined by means of the methods of Functional Analysis (HahnBanach limits) cannot in general be used to replace the more concrete matrix methods of summation (Toeplitz-limits). In the present paper, we propose to use the methods of Non-standard Analysis in order to bridge the gap between the Hahn-Banach-limits on one hand and Toeplits-limits on the other hand. Thus, we shall derive generalized limits which, while clearly related to the matrix methods, are efficient for all bounded sequences. These generalized limits are given by certain linear forms with coefficients in a nonstandard model of analysis, and some of them satisfy also conditions 1.6 and 1.7. More generally, it will be shown that there exist non-standard models of analysis in which all continuous linear functionals on $m$ can be represented by linear forms in a sense which will be made precise in due course.

The foundations of Non-standard Analysis are sketched in Ref. 7 and developed in greater detail in Refs. 8 and 9. The scope of the theory of Ref. 8 is more comprehensive inasmuch as it is based on a higher order language, $L$. We shall adopt $L$ as the basis of the present paper, but in order to follow it, it will be sufficient to suppose that $L$ is some formal language which is appropriate to the predicate calculus of order $\omega$.

As mentioned in Ref. 7, particular non-standard models of analysis are provided by the ultrapowers of the real numbers, $R_{0}$, which constitute proper extensions of $R_{0}$. The ultrapower technique applies both to the lower predicate calculus and to the higher order language which will be considered here. For the notions which can be expressd in the lower predicate calculus, W. A. J. Luxemburg has given a detailed and expert development of Non-standard Analysis in terms of ultrapowers (Ref. 5, compare also Ref. 6). When specific ultrapowers are used, the explicit use of a formal language can be avoided 
and the fact that a non-standard model of analysis has in a definite formal sense the same properties as the standard model remains in the background, as a heuristic principle. This has the advantage of making the subject comprehensible to analysts who are not familiar with the formal languages of Mathematical Logic and also reveals certain aspects of the procedure which are not apparent if the structure of the non-standard model remains unspecified. On the other hand, by using a formal language we may establish the truth of a vast number of useful assertions about the non-standard model simply but rigorously by transfer from the standard case. If no formal language is used we have to prove all these results ab initio.

2. $Q$-sequences. Let $R_{0}$ be the field of real numbers, and let $K_{0}$ be the set of all sentences which are formulated in a higher order language $L$, as in Ref. 8 (compare $\S 1$, above) and which hold in $R_{0}$. Thus, $L$ contains (symbols for) all real numbers, for all $n$-ary relations on real numbers, including unary relations, or sets, and also for concepts of higher type such as relations between sets, relations between relations, etc. In such a language, an infinite sequence $\left\{s_{n}\right\}, n=$ $1,2,3, \cdots$ is represented by a binary relation $\sigma(x, y)$ in which the domain of the first variable is the set of positive integers, such that for every positive integer $n$ there is a unique real number a for which $\sigma(n, a)$ holds in $R_{0}$ (and hence, is a sentence of $K_{0}$ ). The set of all these relations then determines a unary relation in $L, \theta(x)$, say, i.e. $\theta(\sigma)$ holds in $R_{0}$ if and only if $\sigma$ represents an infinite sequence as indicated.

Now let ${ }^{*} R$ be a non-standard model of $K_{0}$, i.e. a model of $K_{0}$ which is different from $R_{0}$ and hence, is a proper extension of $R_{0}$. Then the unary relation of $L$ which determines the set of natural numbers, $N$, in $R_{0}$ determines a non-standard model of the natural numbers, ${ }^{*} N$, in $* R$, and the predicate $\theta(x)$ determines a set of nonstandard infinite sequences in $* R$, i.e. a set of functions whose domain is the set of positive integers in ${ }^{*} R,{ }^{*} N-\{0\}$, and which take values in ${ }^{*} R$. The sequences which are determined in this way are called $Q$-sequences ( $Q$ for quasi-standard). In particular, if $\left\{s_{n}\right\}$ is an infinite sequence in $R_{0}$, which corresponds to a binary relation $\sigma(x, y)$ as above, then $\sigma(x, y)$ determines in ${ }^{*} R$ a $Q$-sequence which coincides with $\left\{s_{n}\right\}$ for all finite values of the subscript $n$, and which will be denoted by $*\left\{s_{n}\right\}$. A $Q$-sequence which is obtained in this way by the extension of a sequence in $R_{0}$ is called an $S$-sequence ( $S$ for standard). For example, the sequence which is given by $s_{n}=1 / n$ for all positive integers, finite or infinite, is an $S$-sequence. There exist $Q$-sequences which are not $S$-sequences. For example, if $\omega$ is a fixed infinite natural number then the sequence which is given by 


$$
\begin{aligned}
& a_{n}=1 / \omega \quad \text { for } \quad 1 \leqq n \leqq \omega \\
& a_{n}=0 \text { for } n>\omega
\end{aligned}
$$

is not an $S$-sequence, since every $S$-sequence takes standard real values for standard (finite) subscripts, but it is a $Q$-sequence, for the assertion,

"For every positive integer $\omega$ there exists an infinite sequence which is given by 2.1 ,"

holds in $R_{0}$, and therefore, can be formulated as a sentence of $K_{0}$ and holds also in ${ }^{*} R$.

More generally, if $\left(a_{k n}\right)$ is a Toeplits-matrix, and $\omega$ is a fixed infinite natural number then the sequence

2.2. $a_{n}=a_{\omega n}$ for all positive integers $n$, finite or infinite, is a $Q$-sequence. It cannot be an $S$-sequence, for 1.1 implies that for every finite positive integer $n, a_{\omega n}$ is infinitesimal. If the sequence were an $S$-sequence then $a_{\omega n}=a_{n}$ would at the same time have to be a standard number and so $a_{n}=0$ for all finite $n$. It is easy to see that the only $S$-sequence which has this property is the zero sequence, $a_{n}=0$ for all $n$, and this contradicts 1.3 , which implies that $\sum_{n=1}^{\infty} a_{\omega n}$ is infinitely close to 1 . In this connection, $\sum_{n=1}^{\infty} a_{\omega n}$ is to be understood in the sense of the classical (Weierstrass) definition.

The equation 2.1 is a special case of 2.2. It is obtained by taking for $\left(a_{k n}\right)$ the matrix of arithmetic means, $a_{k n}=1 / k$ for $n \leqq k, a_{k n}=0$ for $n>k$.

On the other hand it is not difficult to define sequence which are not even $Q$-sequences. For example, the sequence which is given by

$$
\begin{array}{ll}
a_{n}=0 & \text { for all finite positive integers } n, \\
a_{n}=1 & \text { for all infinite positive } n,
\end{array}
$$

cannot be a $Q$-sequence, for if it were, then the set of all infinite natural numbers would be definable in ${ }^{*} R$ as the set of all natural numbers for which $a_{n}=1$. Every nonempty set of natural numbers which is definable in ${ }^{*} R$ must have a first element, for this is a property of subsets of natural numbers which can be formulated as a sentence of $K_{0}$. The set of infinite natural numbers in ${ }^{*} R$ is not empty but does not possess a first element. This shows that 2.3 cannot be a $Q$-sequence.

A more general property of $Q$-sequences, which will be made use. of in the sequel is as follows.

2.3. THEOREM. Let $\left\{A_{n}\right\}$ be a Q-sequence such that $A_{n}$ is infinitesimal for all finite $n$. Then there exists an infinite natural number $\omega$ such that $A_{n}$ is infinitesimal for all $n<\omega$. 
Proof. A $Q$-set is a set which exists in ${ }^{*} R$ as a model of $K_{0}$ (just as a $Q$-sequence is a sequence which exists in ${ }^{*} R$ ). Supposing that $\left\{A_{n}\right\}$ satisfies the assumptions of the theorem, let $B$ be the set of all positive integers in ${ }^{*} R$ such that $n\left|A_{n}\right| \geqq 1$. Then $B$ is a $Q$ set (since it is defined in terms of a $Q$-sequence). If $B$ is empty then $n\left|A_{n}\right|<1$ and so $\left|A_{n}\right|<1 / n$ for all positive integers $n$. This shows in particular that $A_{n}$ is infinitesimal for all infinite $n$ since $1 / n$ is then infinitesimal. We conclude that in this case the conclusion of the theorem is satisfied by all infinite positive integers $\omega$. If $B$ is not empty then it includes a smallest element, $\omega$, for it is a property of every nonempty subset of the natural numbers in $R_{0}$, and hence also in ${ }^{*} R$, to possess a smallest element. Moreover, $\omega$ must be infinite, otherwise $\omega\left|A_{\omega}\right|$ would be infinitesimal and hence, smaller than 1. For $n<\omega$ we have again $n\left|A_{n}\right|<1,\left|A_{n}\right|<1 / n, A_{n}$ is infinitesimal also for all infinite $n$ less than $\omega$. This completes the proof of 2.3 .

Let $\left\{a_{n}\right\}$ be a $Q$-sequence. Then the infinite sum $\sum_{n=1}^{\infty}\left|a_{n}\right|$ may or may not exist in the sense of the classical (Weierstrass) definition, as applied to ${ }^{*} R$. Thus, $\sum_{n=1}^{\infty}\left|a_{n}\right|$ exists if and only if the partial sums $\sum_{n=1}^{k}\left|a_{n}\right|$ is uniformly bounded in $* R$ as $k$ varies over all finite or infinite positive integers. In particular, if $\sum_{n=1}^{\infty}\left|a_{n}\right|$ exists and equals a finite number in ${ }^{*} R$ (i.e. a number of ${ }^{*} R$ which is smaller than some number of $R_{0}$ ) then $\left\{a_{n}\right\}$ will be called an $S$-bounded form. The reason for this terminology will become apparent presently. A $Q$-sequence $\left\{a_{n}\right\}$ is an $S$-bounded form if and only if there exists a standard real number $A$ and that $\sum_{n=1}^{k}\left|a_{n}\right| \leqq A$ for all $k$, finite or infinite.

Let ${ }^{*} m$ be the extension of the space $m$ to ${ }^{*} R$. Thus, ${ }^{*} m$ consists of all $Q$-sequences which are bounded in ${ }^{*} R$. In particular, ${ }^{*} m$ contains all the $S$-sequences ${ }^{*} \sigma$ which are the extensions to ${ }^{*} R$ of bounded sequences $\sigma=\left\{s_{n}\right\}$ in $R_{0}$.

Let $\alpha=\left\{a_{n}\right\}$ be an $S$-bounded from, such that $\sum_{n=1}^{\infty}|a|=A$, where $A$ is a finite number. We shall associate with $\alpha$ a functional $F_{\alpha}(x)$ with domain $m$ and range in $R_{0}$, in the following way. For every $\sigma=\left\{s_{n}\right\}$ in $m$, the sum $\sum_{n=1}^{\infty} a_{n} s_{n}$ exists in ${ }^{*} R$ since, for every finite or infinite positive integer $k$,

$$
\left|\sum_{n=1}^{k} a_{n} s_{n}\right| \leqq \sum_{n=1}^{k}\left|a _ { n } \left\|s _ { n } \left|\leqq\left\|\sigma \sum_{n=1}^{k}\left|a_{n}\right| \leqq\right\| \sigma \| A,\right.\right.\right.
$$

taking into account that $\|\sigma\|$ which is a bound for the absolute values of the element of $\sigma$ must be a bound also for the absolute values of the elements of ${ }^{*} \sigma$. Moreover, (2.4) shows that $\sum_{n=1}^{\infty} a_{n} s_{n}$ is actually finite, since it cannot exceed the finite number $\|\sigma\| A$, and accordingly 
possesses a standard part. Denoting by ${ }^{0} a$ the standard part of any finite $a$, we now put

$$
F_{\alpha}(\sigma)={ }^{0}\left(\sum_{n=1}^{\infty} a_{n} s_{n}\right)
$$

and we claim that $F_{\alpha}(x)$ is a continuous linear functional on $m$.

Indeed, if $\sigma=\left\{s_{n}\right\}$ and $\sigma^{\prime}=\left\{s_{n}^{\prime}\right\}$ are two sequences in $m$ and $\lambda$ is a real number (in $R_{0}$ ) then

$$
\begin{aligned}
F_{\alpha}\left(\sigma+\sigma^{\prime}\right) & =\left(\sum_{n=1}^{\infty} a_{n}\left(s_{n}+s_{n}^{\prime}\right)\right)=\left(\sum_{n=1}^{\infty} a_{n} s_{n}+\sum_{n=1}^{\infty} a_{n} s_{n}^{\prime}\right) \\
& =\left(\sum_{n=1}^{\infty} a_{n} s_{n}\right)+{ }^{0}\left(\sum_{n=1}^{\infty} a_{n} s_{n}^{\prime}\right)=F_{\alpha}(\sigma)+F_{\alpha}\left(\sigma^{\prime}\right)
\end{aligned}
$$

and

$$
F_{\alpha}(\lambda \sigma)={ }^{0}\left(\sum_{n=1}^{\infty} a_{n} \lambda s_{n}\right)={ }^{0}\left(\lambda \sum_{n=1}^{\infty} a_{n} s_{n}\right)=\lambda^{0}\left(\sum_{n=1}^{\infty} a_{n} s_{n}\right)=\lambda F_{\alpha}(\sigma),
$$

showing that the functional is linear. Also, by $2.4,\left|F_{\alpha}(\sigma)\right| \leqq A\|\sigma\|$ showing that $F_{\alpha}(x)$ is continuous and, moreover that the norm of $F_{\alpha}(x)$ does not exceed $A,\left\|F_{\alpha}(x)\right\| \leqq A$.

3. Generalized limits. Let $\alpha$ be an $S$-bounded form, and let $F_{a}(x)$ be the linear functional associated with $\alpha$, as introduced in $\S 2$ above. Then

3.1. THEOREM. In order that $F_{\alpha}(x)$ be a Hahn-Banach limit for a given S-bounded form $\alpha=\left\{a_{n}\right\}$ is necessary and sufficient that

3.2. $a_{n}$ is infinitesimal for all finite $n, a_{n} \simeq 0$, and

3.3. $\sum_{n=1}^{\infty} a_{n}$ is infinitely close to $1, \sum_{n=1}^{\infty} a_{n} \simeq 1$.

Proof. The conditions are necessary. For any finite positive integer $n$, let $\sigma$ be the sequence (in $m$ ) which is defined by

$$
s_{k}=0 \quad \text { for } k \neq n, \quad s_{n}=1 .
$$

Then 3.4 holds also for the extension of $\sigma$ to ${ }^{*} R$, and so

$$
F_{\alpha}(\sigma)={ }^{0}\left(\sum_{k=1}^{\infty} a_{k} s_{k}\right)={ }^{0} a_{n}
$$

where ${ }^{0} a_{n}$ is the standard part of $a_{n}$. On the other hand, since $F_{\alpha}(x)$ is a Hahn-Banach limit, we must have $F_{\alpha}(\sigma)=0$, which is the limit 
of $\sigma$. Hence ${ }^{0} a_{n}=0$, i.e. $a_{n} \simeq 0, a_{n}$ is infinitesimal.

Again, let $\sigma$ be the sequence in which is defined by

$$
s_{n}=1 \text { for all } n \text {. }
$$

Then the limit of $\sigma$ is 1 , and $s_{n}=1$ also for all infinite values of $n$. Hence, $F_{\alpha}(\sigma)={ }^{0}\left(\sum_{n=1}^{\infty} s_{n}\right)=1$, and so $\sum_{n=1}^{\infty} \simeq 1$, proving 3.3.

The conditions are also sufficient. For any positive integer $n$, put $A_{n}=\sum_{k=1}^{n}\left|a_{k}\right|$. Then 3.2 implies that $A_{n}$ is infinitesimal for all finite $n$. Hence, by 2.3 , there exists an infinite integer $\omega$ such that $A_{\omega-1}=$ $\sum_{k=1}^{\omega-1}\left|a_{k}\right|=\eta$, say, is infinitesimal. On the other hand, since the sum $\sum_{n=1}^{\infty}\left|a_{n}\right|$ exists, there is an infinite integer $\Omega>\omega$, such that $\sum_{n=\Omega}^{\infty}\left|a_{n}\right| \leqq$ $\eta$. Referring to 3.3 we may then conclude that

$$
\sum_{n=\omega}^{8} a_{n} \simeq 1
$$

or, which is the same, that

$$
\sum_{n=\omega}^{2} a_{n}=1-\varepsilon
$$

where $\varepsilon$ is infinitesimal (positive, or negative, or zero).

Now let $\sigma=\left\{s_{n}\right\}$ be any convergent sequence in $m$ (i.e. in $R_{0}$ ), with limit $s$. Then $s-s_{n}$ is infinitesimal for all infinite $n$. Let $\theta$ be the lowest upper bound of the elements of the set $T$ of all numbers $\left|s-s_{n}\right|$ for $n \geqq \omega$. $\theta$ exists since $T$ is a bounded $Q$-set, and the fact that every bounded set has a lowest upper bound transfers from $R_{0}$ to ${ }^{*} R$ since it can be expressed as a sentence of $K_{0}$. But the elements of $T$ are all infinitesimal, and so $\theta$ also must be infinitesimal (possibly zero). For if $\tau$ is an upper bound for $T$, and $\tau$ is not infinitesimal then $\frac{1}{2} \tau$ also is an upper bound for $T$ and so $\tau$ cannot be the lowest upper bound of $T$.

We have to show that

$$
s=F_{a}(\sigma)={ }^{0}\left(\sum_{n=1}^{\infty} a_{n} s_{n}\right)
$$

or, which is the same that $\left|s-\sum_{n=1}^{\infty} a_{n} s_{n}\right|$ is infinitesimal.

Now, computing in ${ }^{*} R$,

$$
\begin{aligned}
\left|s-\sum_{n=1}^{\infty} a_{n} s_{n}\right| & =\left|\left(\sum_{n=\omega}^{\Omega} a_{n}+\varepsilon\right) s-\sum_{n=1}^{\infty} a_{n} s_{n}\right| \\
& =\left|\varepsilon s-\sum_{n=1}^{\omega-1} a_{n} s_{n}+\sum_{n=\omega}^{2-1} a_{n}\left(s-s_{n}\right)-\sum_{n=\Omega}^{\infty} a_{n} s_{n}\right| \\
& \leqq|\varepsilon||s|+\sum_{n=1}^{\omega-1}\left|a_{n}\right|\left|s_{n}\right|+\sum_{n=\omega}^{\Omega-1}\left|a_{n}\right|\left|s-s_{n}\right|+\sum_{n=0}^{\infty}\left|a_{n}\right|\left|s_{n}\right| \\
& \leqq|\varepsilon||s|+\eta|| \sigma\|+A \theta+\eta|| \sigma\| \leqq(|\varepsilon|+2 \eta)\|\sigma\|+A \theta
\end{aligned}
$$


where the sum on the right hand side is indeed infinitesimal. This completes the proof of 3.1 .

Let $\left(a_{k n}\right)$ be any Toeplitz-matrix in the ordinary sense, i.e. in $R_{0}$, and let $\omega$ be an infinite positive integer. Then the sequence $\alpha=\left\{a_{n}\right\}$ which is given by 2.2 is an $S$-bounded form since, by 1.2 ,

$$
\sum_{n=1}^{\infty}\left|a_{n}\right|=\sum_{n=1}^{\infty}\left|a_{\omega n}\right|<M .
$$

At the same time, $\alpha$ satisfies 3.2 and 3.3 by 1.1 and 1.3 respectively. It follows that $F_{\alpha}(x)$ is a generalized limit.

If we wish to ensure that an $S$-bounded form defines a BanachMazur limit we require an additional condition.

3.6. THeOREM. Let $\alpha=\left\{a_{n}\right\}$ be an S-bounded form which satisfies 3.2 and 3.3 such that $a_{n} \geqq 0$ for all $n$ and such that

$$
\sum_{n=1}^{\infty}\left|a_{n+1}-a_{n}\right|
$$

is infinitesimal. Then $F_{a}(x)$ is a Banach-Mazur limit.

Proof. We know from 3.1 that $F_{\alpha}(x)$ is at any rate a Hahn-Banach limit. Thus, it only remains for us to show that $F_{a}(x)$ satisfies condition 1.7, for any convergent sequence $\sigma=\left\{s_{n}\right\}$ in $m$. Computing in $* R$, we have in fact

$$
\begin{aligned}
\left|\sum_{n=1}^{\infty} a_{n} s_{n}-\sum_{n=1}^{\infty} a_{n} s_{n+1}\right| & =\left|a_{1} s_{1}+\sum_{n=1}^{\infty}\left(a_{n+1}-a_{n}\right) s_{n+1}\right| \\
& \leqq\left|a_{1}\right|\|\sigma\|+\left(\sum_{n=1}^{\infty}\left|a_{n+1}-a_{n}\right|\right)\|\sigma\|,
\end{aligned}
$$

where the right hand side is infinitesimal, by 3.2 and 3.7. Hence,

$$
\sum_{n=1}^{\infty} a_{n} s_{n} \simeq \sum_{n=1}^{\infty} a_{n} s_{n+1}
$$

and so

$$
F_{a}\left(\left\{s_{n}\right\}\right)={ }^{0}\left(\sum_{n=1}^{\infty} a_{n} s_{n}\right)={ }^{0}\left(\sum_{n=1}^{\infty} a_{n} s_{n+1}\right)=F_{\alpha}\left(\left\{s_{n+1}\right\}\right),
$$

showing that 1.7 is satisfied. The truth of 1.6 is obvious, proving 3.6.

If $a_{n}=a_{\omega n}$, for infinite $\omega$, where $\left(a_{k n}\right)$ is a Toeplitz-matrix as above then 3.7 is satisfied provided

$$
\lim _{k \rightarrow \infty}\left|a_{k, n+1}-a_{k n}\right|=0
$$

in the standard sense, i.e. in $R_{0}$, and $a_{n} \geqq 0$ for all $n$, provided the 
elements of the matrix are nonnegative.

A particular example of a sequence $\left\{a_{n}\right\}$ which provides a BanachMazur limit is given by 2.1 .

4. Representation of continuous linear functionals on $m$ in nonstandard analysis. We have seen that every $S$-bounded form in a non-standard model of analysis gives rise to a continuous linear functional on $m$. Conversely, we may ask whether every continuous linear functional on $m$ can be represented in this way. In this direction, we have the following rather strong result, whose proof is the main purpose of this section.

4.1. THEOREM. There exists a non-standard model of analysis, ${ }^{*} R$, such that for every continuous linear functional $F(x)$ on $m$, there is an S-bounded form $\alpha$ in ${ }^{*} R$ such that $F(x) \equiv F_{\alpha}(x)$ identically on $m$.

In order to prove 4.1, we require some auxiliary considerations which refer to the standard case (i.e. to $R_{0}$ ).

Let $\sigma^{1}, \cdots, \sigma^{\nu}$ be any finite sequence of elements of $m$, where

$$
\sigma^{i}=\left\{s_{1}^{i}, s_{2}^{i}, \cdots, s_{n}^{i}, \cdots\right\}, \quad i=1, \cdots, \nu
$$

and let $\varepsilon$ be positive, otherwise arbitrary. Then (compare Ref. 1, p. 69) there exists a positive integer $\mu$, such that for every set of real numbers $\lambda_{1}, \cdots, \lambda_{\nu}$,

$$
\left\|\lambda_{1} \sigma^{1}+\lambda_{2} \sigma^{2}+\cdots+\lambda_{\nu} \sigma^{\nu}\right\| \leqq \max _{1 \leqq i \leqq \mu}\left|\lambda_{1} s_{i}^{1}+\lambda_{2} s_{i}^{2}+\cdots+\lambda_{\nu} s_{i}^{\nu}\right|(1+\varepsilon) .
$$

It follows that if we define the $\mu$-dimensional vectors $\tau^{i}, \mathbf{i}=1, \cdots, \nu$, by

$$
\tau^{i}=\left(s_{1}^{i}, s_{2}^{i}, \cdots, s_{\mu}^{i}\right)
$$

and use the sup (lowest upper bound) norm in the space $m^{\prime}$ spanned by these vectors, then for every set of real numbers $\lambda_{1}, \cdots, \lambda_{\nu}$

$$
\left\|\lambda_{1} \sigma^{1}+\lambda_{2} \sigma^{2}+\cdots+\lambda_{\nu} \sigma^{\nu}\right\| \leqq(1+\varepsilon)\left\|\lambda_{1} \tau^{1}+\lambda_{2} \tau^{2}+\cdots+\lambda_{v} \tau^{\nu}\right\| .
$$

Hence, for any continuous linear functional $F(x)$ on $m$,

$$
\begin{aligned}
\left|F\left(\lambda_{1} \sigma^{1}+\cdots+\lambda_{\nu} \sigma^{\nu}\right)\right| & \leqq\|F(x)\|\left\|\lambda_{1} \sigma^{1}+\cdots+\lambda_{\nu} \sigma^{\nu}\right\| \\
& \leqq(1+\varepsilon)\|F(x)\|\left\|\lambda_{1} \tau^{1}+\cdots+\lambda_{\nu} \tau^{\nu}\right\|
\end{aligned}
$$

which is equivalent to

$$
\left|\lambda_{1} F\left(\sigma^{1}\right)+\cdots+\lambda_{2} F\left(\sigma^{\nu}\right)\right| \leqq(1+\varepsilon)\|F(x)\|\left\|\lambda_{1} \tau^{1}+\cdots+\lambda_{\nu} \tau^{\nu}\right\| .
$$


In $m^{\prime}$, define a functional $G(x)$ by

$$
G\left(\lambda_{1} \tau^{1}+\cdots+\lambda_{\nu} \tau^{\nu}\right)=\lambda_{1} F\left(\sigma^{1}\right)+\cdots+\lambda_{\nu} F\left(\sigma^{\nu}\right) .
$$

This definition is unique, for 4.2 shows that if two representations of the form $\lambda_{1} \tau^{1}+\cdots+\lambda_{\nu} \tau^{\nu}$ coincide, then the corresponding expressions on the right hand side of 4.3 coincide. Moreover, by $4.3, G(x)$ is clearly additive, and by 4.2 it is also continuous, with norm $\|G(x)\| \leqq$ $(1+\varepsilon)\|F(x)\|$ (where the norm of $F$ and $G$ refer to the spaces $m$ and $m^{\prime}$ respectively).

Now let $m_{\mu}$ be the full $\mu$-dimensional real space under the sup norm, so that $m^{\prime}$ is a subspace of $m_{\mu}$. Extend $G(x)$ to all of $m_{\mu}$ without increasing its norm and, without fear of confusion, call the result again $G(x)$. Let $\xi^{i}=\left(\delta_{i 1}, \delta_{i 2}, \cdots, \delta_{i \mu}\right), i=1, \cdots, \mu$, where $\delta_{i k}$ is the Kronecker delta, and put $a_{i}=G\left(\xi^{i}\right)$. Let

$$
\xi^{0}=\xi^{1} s g a_{1}+\xi^{2} s g a_{2}+\cdots+\xi^{\mu} s g a_{\mu}
$$

(where $\operatorname{sg} a$ is $-1,0$, or 1 according $a$ is negative, zero, or positive) then $\left\|\xi^{0}\right\|=1$ provided at least one of the $a_{i}$ is different from zero. Also,

$$
\begin{aligned}
G\left(\xi^{0}\right) & =\left|a_{1}\right|+\left|a_{2}\right|+\cdots+\left|a_{\mu}\right| \leqq\|G(x)\|\left\|\xi^{0}\right\| \\
& \leqq(1+\varepsilon)\|F(x)\| \mid \xi^{0} \|
\end{aligned}
$$

and so, in any case

$$
\left|a_{1}\right|+\left|a_{2}\right|+\cdots+\left|a_{3}\right| \leqq(1+\varepsilon)|| F(x) \| \text {. }
$$

Now

$$
\tau^{i}=s_{1}^{i} \xi^{1}+s_{2}^{i} \xi^{2}+\cdots+s_{\mu}^{i} \xi^{\mu}
$$

and so

$$
F\left(\sigma^{i}\right)=G\left(\tau^{i}\right)=a_{1} s_{1}^{i}+a_{2} s_{2}^{i}+\cdots+a_{\mu} s_{\mu}^{i} .
$$

Summing up, we have established (compare Ref. 1, p. 70).

4.4. THEOREM. Let $F(x)$ be a continuous linear functional on $m$, let $\varepsilon>0$, and let $\sigma^{i}=\left\{s_{n}^{i}\right\}$ be arbitrary elements of $m, \varepsilon=1, \cdots, \nu$. Then there exist real numbers $a_{1}, \cdots, a_{\mu}$, such that

$$
a_{1} s_{1}^{i}+a_{2} s_{2}^{i}+\cdots+a_{\mu} s_{\mu}^{i}=F\left(\sigma_{i}\right), \quad i=1, \cdots, \nu
$$

while

$$
a_{1}|+| a_{2}|+\cdots+| a_{\mu}|\leqq(1+\varepsilon)| \mid F(x) \| \cdot
$$

We are now in a position to prove 4.1. 
Consider the statement:

" $x$ is an absolutely convergent sequence, $x=\left\{x_{1}, x_{2}, \cdots\right\}$ such that $\sum_{n=1}^{\infty}\left|x_{n}\right|$ does not exceed $y$, and $z$ is a bounded sequence, $z=\left\{z_{1}, z_{2}, z_{3}, \cdots\right\}$, such that $\sum_{n=1}^{\infty} x_{n} z_{n}$ is equal to $w . "$

This may be formulated in $L$ as a predicate $Q(x, y, z, w)$ whose extralogical constants occur in $K_{0}$. We now extend our vocabulary by introducing for each bounded linear functional $F(x)$ on $m$ a constant $a_{F}$, different $a_{F}$ being used for different functionals. For any continuous linear functional $F(x)$ on $m$, and for any $\sigma$ in $M$, we define a sequence of formal sentences $Y_{n}(F, \sigma)$ by

$$
Y_{n}(F, \sigma)=Q\left(a_{F},\left(1+\frac{1}{n}\right)\|F(x)\|, \sigma, F(\sigma)\right) \quad n=1,2, \cdots .
$$

Let $K_{1}$ be the set of all sentences which are obtained in this way. We claim that the set $H=K_{0} \cup K_{1}$ is consistent. In order to establish this fact it is sufficient to show that $H^{\prime}=K_{0} \cup K_{1}^{\prime}$ is consistent where $K_{1}^{\prime}$ is an arbitrary finite subset of $K_{1}$. We may limit the class of $K_{1}^{\prime}$ to be considered somewhat by observing that for any $F$ and $\sigma$,

$$
Y_{n}(F, \sigma) \supset Y_{m}(F, \sigma)
$$

is deducible from $K_{0}$ provided $n>m$. This implies that we may suppose the subscripts of the sentences which belong to the given $K_{1}^{\prime}$ to be all equal. Indeed, if this is not the case from the outset we replace them all by the greatest subscripts which occurs in $K_{1}^{\prime}$ to begin with.

Suppose in accordance with this remark that $K_{1}^{\prime}$ consists of the sentences

$$
\begin{aligned}
& Y_{n}\left(F_{1}, \sigma^{1}\right), Y_{n}\left(F_{1}, \sigma^{2}\right), \cdots, Y_{n}\left(F_{1}, \sigma^{\nu_{1}}\right) \\
& Y_{n}\left(F_{2}, \sigma^{\nu_{1}+1}\right), Y_{n}\left(F_{2}, \sigma^{\nu_{1}+2}\right), \cdots, Y_{n}\left(F_{2}, \sigma^{\nu_{2}}\right) \\
& \quad \ldots \ldots \ldots \cdots \cdots \cdots \cdots \cdots \cdots \cdots \cdots \cdots \cdots \cdots \cdots \cdots \cdots \\
& Y_{n}\left(F_{k}, \sigma^{\nu_{k-1}+1}\right), Y_{n}\left(F_{k}, \sigma^{\nu_{k-1}+2}\right), \cdots, Y_{n}\left(F_{k}, \sigma^{\nu_{k}}\right) .
\end{aligned}
$$

In order to prove that $H^{\prime}$ is consistent we shall show that, with a suitable interpretation of the constants $a_{F_{1}}, \cdots, a_{F_{k}}, R_{0}$ becomes a model of $H^{\prime}$. It is in fact evident that $R_{0}$ is a model of $K_{0}$. Interpreting $a_{F_{1}}$ as the infinite sequence

$$
\left\{a_{1}, \cdots, a_{\mu}, 0,0,0, \cdots\right\}
$$

for a suitable set of real numbers $\left(a_{1}, \cdots, a_{\mu}\right)$ such as exists according 
to Theorem 4.4, if we put $\varepsilon=1 / n$ and identify $F$ with $F_{1}$, and $\sigma^{1}, \cdots, \sigma^{\nu}$ with the present $\sigma^{1}, \cdots, \sigma^{\nu_{1}}$ respectively, we find that the sentences in the first line of 4.8 also holds in $R_{0}$. A similar procedure shows that, with the appropriate interpretation, the remaining sentences of 4.8 also hold in $R_{0}$. This shows that $H^{\prime}$ is consistent and hence, that $H$ is consistent.

Let $* R$ be a model of $H$. For any continuous linear functional on $m, F(x)$, the constant $a_{F}$ denotes an infinite sequence

$$
\alpha=\left\{a_{1}, a_{2}, a_{3}, \cdots, a_{k}, \cdots\right\}
$$

(where the subscript varies over the positive integers in ${ }^{*} R$ ) such that for any $\sigma=\left\{s_{k}\right\}$ in $m$,

$$
\sum_{k=1}^{\infty} a_{k} s_{k}=F(\sigma) .
$$

Moreover, for any finite positive integer $n$,

$$
\sum_{k=1}^{\infty}\left|a_{k}\right| \leqq\left(1+\frac{1}{n}\right)\|F(x)\| \text {. }
$$

Indeed, the validity of 4.9 and 4.10 is asserted by $Y_{n}(F, \sigma)$, and this sentence holds in ${ }^{*} R$. Since $n$ is a positive and finite integer, but otherwise arbitrary, we may conclude from 4.10 that

$$
{ }^{0}\left(\sum_{k=1}^{\infty}\left|a_{k}\right|\right) \leqq\|F(x)\|
$$

so that $a$ is an $S$-bounded form. Equation 4.9 shows that $F_{\alpha}(x) \equiv$ $F(x)$ identically on $m$ since it implies the weaker relation ${ }^{0}\left(\sum_{k=1}^{\infty} a_{k} s_{k}\right)=$ $F(\sigma) . \quad{ }^{*} R$ is a model of $K_{0}$ but it cannot be the standard model, $R_{0}$, for it is not true that every continuous linear functional in ${ }^{\circ} R$ can be represented as in 4.9. This completes the proof of 4.1.

Suppose now that we have strict inequality in 4.11 , then there exists a standard number $M, 0<M<\|F(x)\|$ such that

$$
\sum_{k=1}^{\infty}\left|a_{k}\right| \leqq M \text {. }
$$

Hence, by 4.9 ,

$$
|F(\sigma)|=\left|\sum_{k=1}^{\infty} a_{k} s_{k}\right| \leqq\|\sigma\| \sum_{k=1}^{\infty}\left|a_{k}\right| \leqq\|\sigma\| M
$$

for all $\sigma$ in $m$. But this contradicts the definition of $\|F(x)\|$ and shows that 4.11 may be replaced by

$$
\left.\sum_{k=1}^{\infty}\left|a_{k}\right|\right)=\|F(x)\| \text {. }
$$


We also observe that the sequences which correspond to the $a_{F_{i}}$ in the consistency proof for $H^{\prime}$ are all finite, i.e. they take the value zero for sufficiently large subscripts. We may therefore add this as a requirement for the sequences $\alpha=\left\{a_{1}, a_{2}, \cdots, a_{k}, \cdots\right\}$ and we may attain in this way that every $\alpha$ is $Q$-finite i.e. that $\alpha_{k}$ is equal to zero for subscripts greater than some $\nu$ which depends on $\alpha$ and which may be infinite. Our remarks are summed up in the following corollary.

4.13. CoROllary to 4.1. In 4.1, we may add the three conditions that the sequences $\alpha$ are finite, that $\sum_{k=1}^{\infty} a_{k} s_{k}=F(\sigma)$ for every $\sigma$ in $m$-and not only ${ }^{\circ}\left(\sum_{k=1}^{\infty} a_{k} s_{k}\right)=F(\sigma)$-and that ${ }^{\circ}\left(\sum_{k=1}^{\infty}\left|a_{k}\right|\right)=$ $\|F(x)\|$.

5. Representation of continuous linear functionals on $m$ by ultrafilter limits. In order to be able to follow the contents of this section, the reader should have some familiarity with the theory of ultrapowers. He will then see that the non-standard model of analysis constructed in the preceding section may be obtained as an ultrapower of $R_{0}$. Let $J=\{r\}$ be the index set of the ultrapower and let $D$ be the ultrafilter on $J$ which determines ${ }^{*} R$. An infinite sequence in ${ }^{*} R$ then is an equivalence class, with respect to $D$, of sets of infinite sequences $\sigma^{\nu}=\left\{s_{1}^{\nu}, s_{2}^{\nu}, \cdots\right\}$ which are indexed in $J$.

A set of real numbers $a_{\nu}$ which is indexed in $J$ tends to the limit $a$ in the ultrafilter $D$, and we write

$$
\lim _{J} a_{\nu}=a(D),
$$

if for every positive $\varepsilon$, the set $\left\{\nu|| a-a_{\nu} \mid<\varepsilon\right\}$ belongs to $D$. With this notation, we have the following "translation" of 4.1 , taking into account 4.13 .

5.1. THEOREM. There exists an index set $J=\{\nu\}$ and an ultrafilter $D$ on $J$ such that-

for every continuous linear functional $F(x)$ on the space $m$ there exists a set of sequences $a^{\nu}=\left\{a_{1}^{\nu}, a_{2}^{\nu}, \cdots\right\}$ which is indexed by $J$ and which satisfies the following conditions.

5.2. The sequences $a^{\nu}$ are finite in the sense that $a_{n}^{\nu}=0$ for all $\nu$ greater than some positive integer $k_{\nu}$,

$$
\lim \sum_{n=1}^{k_{\nu}}\left|a_{n}\right|=\|F(x)\|
$$

and

5.4. for any $\sigma=\left\{s_{1}, s_{2}, \cdots\right\}$ in $m$, 


$$
\lim _{J} \sum_{n=1}^{k_{\nu}} a_{n} s_{n}=F(\sigma)(D)
$$

Moreover, in view of 4.9 we may replace 5.4 by the condition

5.5. for any $\sigma$ in $m$, the set

$$
\left\{\nu \mid \sum_{n=1}^{k_{\nu}} a_{n} s_{n}=F(\sigma)\right\}
$$

belongs to $D$ -

which is somewhat stronger than 5.4.

Theorem 5.1 is not only an immediate consequence of 4.1 and 4.13, but conversely it implies those results as we may see by taking as the required non-standard model of analysis the ultrapower $\left(R_{0}\right)_{D}^{J}$, i.e. the direct product of a set of copies of $R_{0}$ which are indexed in $J$, reduced with respect to the ultrafilter $D$. Theorem 5.1 has the "advantage" that it does not involve the notion of a non-standard model of analysis but it lacks the intuitive significance of the preceeding theory.

Except for the requirement of positivity in the definition of the Banach-Mazur limit all our concepts and results carry over to the complex case. The proofs also remain applicable with minor modifications. In particular, in $\S 4.4 s g a$ has to be replaced by $e^{-i \arg a}$ for $a \neq 0$.

The reader may find it interesting to compare 5.1 above with the representation of continuous linear functionals on $m$ by Moore-Smith limits, which is due to Hildebrandt (Ref. 4) and with the matrix representation due to Mazur (compare Ref. 1, p. 72) which, however, applies only to separable subspaces of $m$.

In conclusion the author is pleased to acknowledge that his thinking on the subject of the present paper has been stimulated by conversations with P. Katz, W. A. J. Luxemburg, A. Meir, and D. Scott.

\section{REFERENCES}

1. S. Banach, Théorie des opérations linéaires, Warsaw, 1932.

2. R. G. Cooke, Infinite matrices and sequence spaces, London, 1950.

3. N. Dunford and J. T. Schwartz, Linear operators, part I: general theory, New York, 1958.

4. T. H. Hildebrandt, On bounded functional operations, Trans. Amer. Math. Soc., 36 (1934), 868-875.

5. W. A. J. Luxemburg, Non-standard Analysis, lecture notes, California Institute of Technology, 1962.

6. - Two applications of the method of construction by ultrapowers to Analysis, Bull. Amer. Math. Soc., 68 (1962), 316-419. 
7. A. Robinson, Non-standard Analysis, Proc. Royal Acad. Sc., Amsterdam, series A, 64 (1961), 423-440.

8. Complex function theory over non-archimedean fields, Technical (scientific) note no. 30, U.S. Air Force contract No. 61 (052)-187, Jerusalem, 1962.

9. - Introduction to model theory and to the metamathematics of algebra, Amsterdam, 1963.

10. R. Sikorski, On the existence of the generalized limit, Studia Mathematicae 12 (1951), 117-124.

UNIVERSity of CALIFornia, Los ANGeles 



\section{PACIFIC JOURNAL OF MATHEMATICS}

\section{EDITORS}

Robert Osserman

Stanford University

Stanford, California

M. G. Arsove

University of Washington

Seattle 5 , Washington
J. DugundjI

University of Southern Califorma: Los Angeles 7, California

Lowell J. Paige

University of California

Los Angeles 24, California

\section{ASSOCIATE EDITORS}

E. F. BECKENBACH

B. H. NeumanN

F. WOLF

K. YosIDA

\section{SUPPORTING INSTITUTIONS}

UNIVERSITY OF BRITISH COLUMBIA

CALIFORNIA INSTITUTE OF TECHNOLOGY

UNIVERSITY OF CALIFORNIA

MONTANA STATE UNIVERSITY

UNIVERSITY OF NEVADA

NEW MEXICO STATE UNIVERSITY

OREGON STATE UNIVERSITY

UNIVERSITY OF OREGON

OSAKA UNIVERSITY

UNIVERSITY OF SOUTHERN CALIFORNIA
STANFORD UNIVERSITY

UNIVERSITY OF TOKYO

UNIVERSITY OF UTAH

WASHINGTON STATE UNIVERSITY

UNIVERSITY OF WASHINGTON

AMERICAN MATHEMATICAL SOCIETY CALIFORNIA RESEARCH CORPORATION SPACE TECHNOLOGY LABORATORIES NAVAL ORDNANCE TEST STATION 


\section{Pacific Journal of Mathematics}

\section{Vol. 14, No. 1 \\ May, 1964}

Richard Arens, Normal form for a Pfaffian .........................

Charles Vernon Coffman, Non-linear differential equations on cones in Banach

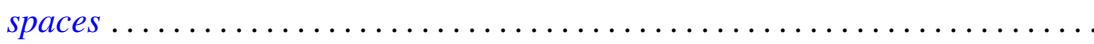

Ralph DeMarr, Order convergence in linear topological spaces ..............

Peter Larkin Duren, On the spectrum of a Toeplitz operator ................

Robert E. Edwards, Endomorphisms of function-spaces which leave stable all

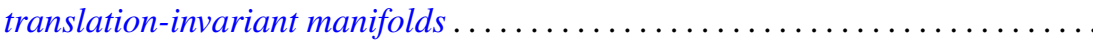

Erik Maurice Ellentuck, Infinite products of isols . . . . . . . . . . . . . . . . 49

William James Firey, Some applications of means of convex bodies . . . . . . . . 53

Haim Gaifman, Concerning measures on Boolean algebras ............. 61

Richard Carl Gilbert, Extremal spectral functions of a symmetric operator. . . . . . 75

Ronald Lewis Graham, On finite sums of reciprocals of distinct nth powers ..... 85

Hwa Suk Hahn, On the relative growth of differences of partition functions ...... 93

Isidore Isaac Hirschman, Jr., Extreme eigen values of Toeplitz forms associated

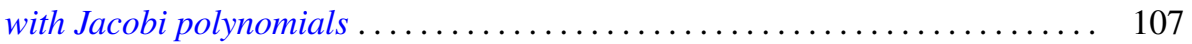

Chen-jung Hsu, Remarks on certain almost product spaces . . . . . . . . . . . 163

George Seth Innis, Jr., Some reproducing kernels for the unit disk . . . . . . . . . 177

Ronald Jacobowitz, Multiplicativity of the local Hilbert symbol . . . . . . . . . . . 187

Paul Joseph Kelly, On some mappings related to graphs ................. 191

William A. Kirk, On curvature of a metric space at a point . . . . . . . . . . . . 195

G. J. Kurowski, On the convergence of semi-discrete analytic functions . . . . . . . 199

Richard George Laatsch, Extensions of subadditive functions . . . . . . . . . . . 209

V. Marić, On some properties of solutions of $\Delta \psi+A\left(r^{2}\right) X \nabla \psi+C\left(r^{2}\right) \psi=0 \ldots 217$

William H. Mills, Polynomials with minimal value sets . . . . . . . . . . . 225

George James Minty, Jr., On the monotonicity of the gradient of a convex

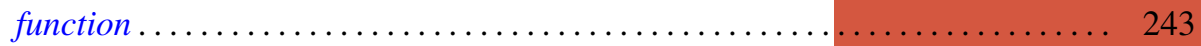

George James Minty, Jr., On the solvability of nonlinear functional equations of 'monotonic' type ................................... 249

J. B. Muskat, On the solvability of $x^{e} \equiv e(\bmod p) \ldots \ldots \ldots \ldots \ldots \ldots \ldots \ldots . \ldots \ldots$

Zeev Nehari, On an inequality of $P . R$. Bessack ................... 261

Raymond Moos Redheffer and Ernst Gabor Straus, Degenerate elliptic

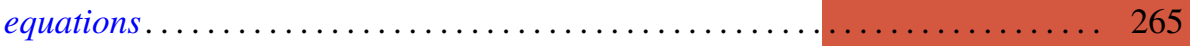

Abraham Robinson, On generalized limits and linear functionals . . . . . . . . . 269

Bernard W. Roos, On a class of singular second order differential equations with a

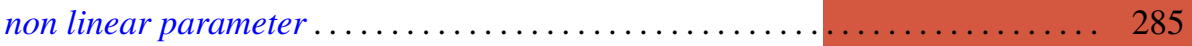

Tôru Saitô, Ordered completely regular semigroups . . . . . . . . . . . . . . . . 295

Edward Silverman, A problem of least area ....................... 309

Robert C. Sine, Spectral decomposition of a class of operators . . . . . . . . . 333

Jonathan Dean Swift, Chains and graphs of Ostrom planes . . . . . . . . . . . 353

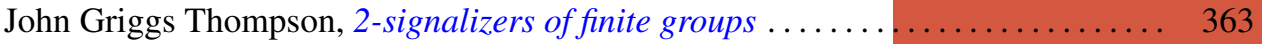

Harold Widom, On the spectrum of a Toeplitz operator . . . . . . . . . . . . . 365 